\title{
Propuesta para una nueva interpretación de los patios de Córdoba (España): paisaje interior, emoción y memoria
}

\author{
Rosa Colmenarejo Fernández \\ Profesora doutora da Universidad Loyola Andalucía, España \\ rcolmenarejo@uloyola.es
}

Resumen La inclusión de la "Fiesta de los Patios de Córdoba" en la Lista Representativa del Patrimonio Cultural Inmaterial de la UNESCO en 2012, ha supuesto el reconocimiento de los valores que ésta aporta tanto a la convivencia y la solidaridad humanas, como al patrimonio natural y cultural de la ciudad. Este artículo incluye una completa revisión bibliográfica sobre el estado de la cuestión, y propone nuevas categorías tipológicas que pretenden contribuir a una actualización de su valoración patrimonial mediante una geografia de las emociones basada en el paseo, el reconocimiento, la identidad y la memoria de sus habitantes, que vendría a incorporarse a las clasificaciones actuales basadas en la arquitectura de lo construido.

Palabras clave: Patrimonio Inmaterial, Fiesta de los Patios de Córdoba, Paisaje interior, Emociones, Lugar de memoria.

\section{INTRODUCCIÓN}

La Convención para la Salvaguardia del Patrimonio Inmaterial (CSPI) establece que el patrimonio cultural inmaterial se constituye por los "usos, representaciones, expresiones, conocimientos y técnicas -junto con los instrumentos, objetos, artefactos y espacios culturales que les son inherentes- que las comunidades, los grupos y en algunos casos los individuos reconozcan como parte integrante de su patrimonio cultural" (UNESCO, 2003). Según la CSPI el patrimonio cultural inmaterial infunde un sentimiento de identidad, se transmite de generación en generación y es recreado constantemente por las comunidades. 
Podría establecerse que la "Fiesta de los patios de Córdoba" (FPC) surge de un hábitat, las casas patio populares o de vecinos que describiremos con detalle a continuación, y un habitus o forma de vida de quienes habitan dichas casas patio. La estratégica combinación de ambos tipos ha generado una dinámica cultural que fue institucionalizada en 1921 por el Ayuntamiento de Córdoba al convocar un Concurso de Patios Populares (CPP) que se mantiene hasta la actualidad. Casas, habitantes y concurso conforman el ser actual de la FPC. La "fiesta" se desarrolla durante doce días del mes de mayo. Las casaspatio seleccionadas a concurso abren sus puertas a visitantes locales y foráneos para que puedan admirar el espacio adornado con flores, macetas, fuentes y elementos decorativos representativos de cómo era la vida en el patio en tiempos de las generaciones anteriores: fotografias, aperos de la vida cotidiana, cacharros de cocinas, etc.

La inclusión de la FPC en la Lista Representativa del Patrimonio Cultural Inmaterial de la Humanidad supone el cumplimiento de los cinco criterios exigidos por la CSPI (UNESCO, 2012). Comprender el alcance del primero de ellos es el objeto de este trabajo:

La FPC constituye un patrimonio inmaterial, atendiendo a la definición del Art. 2 de la Convención: es un evento festivo comunitario que proporciona un sentido de identidad y continuidad a los habitantes de Córdoba, que reconocen esta expresión como un componente importante de su patrimonio cultural inmaterial;

Las casas-patio de Córdoba (España), soporte espacial de la FPC, son un patrimonio muy atomizado, de propiedad privada, heterogéneo en su tipología y dimensión, y muy dependiente de las decisiones y acciones de sus propietarios o habitantes. En un estudio realizado en 2012 por Esteban y Obrero ${ }^{1}$ se ha constatado que de los 405 inmuebles, casas patio, casas de vecinos, corrales, casas de paso, de los que se ha tenido alguna noticia de su existencia en los últimos 50 años, aún quedan en pie 276. Estos bienes muebles son los espacios culturales que dan soporte al Concurso de Patios Populares (CPP) que organiza el ayuntamiento de Córdoba anualmente desde 1933, y que conlleva la apertura al público de éstos durante un período de doce días en el mes de mayo. El foco de atracción turística que implica esta actividad ha supuesto un incremento paulatino de visitas, al tiempo que el tipo de patios que solicitaban su inscripción en el concurso sufría considerables variaciones.

1 Cfr. "Córdoba ha perdido casi un tercio de sus patios en las últimas décadas", Cordópolis, 8 de diciembre de 2012: < http:// cordopolis.es/cordoba-ha-perdido-casi-un-tercio-de-sus-patios-en-las-ultimas-decadas/>. Accedido 18 de noviembre de 2013. 
Figura 1 - Plano Situación Centro Histórico de Córdoba (España)

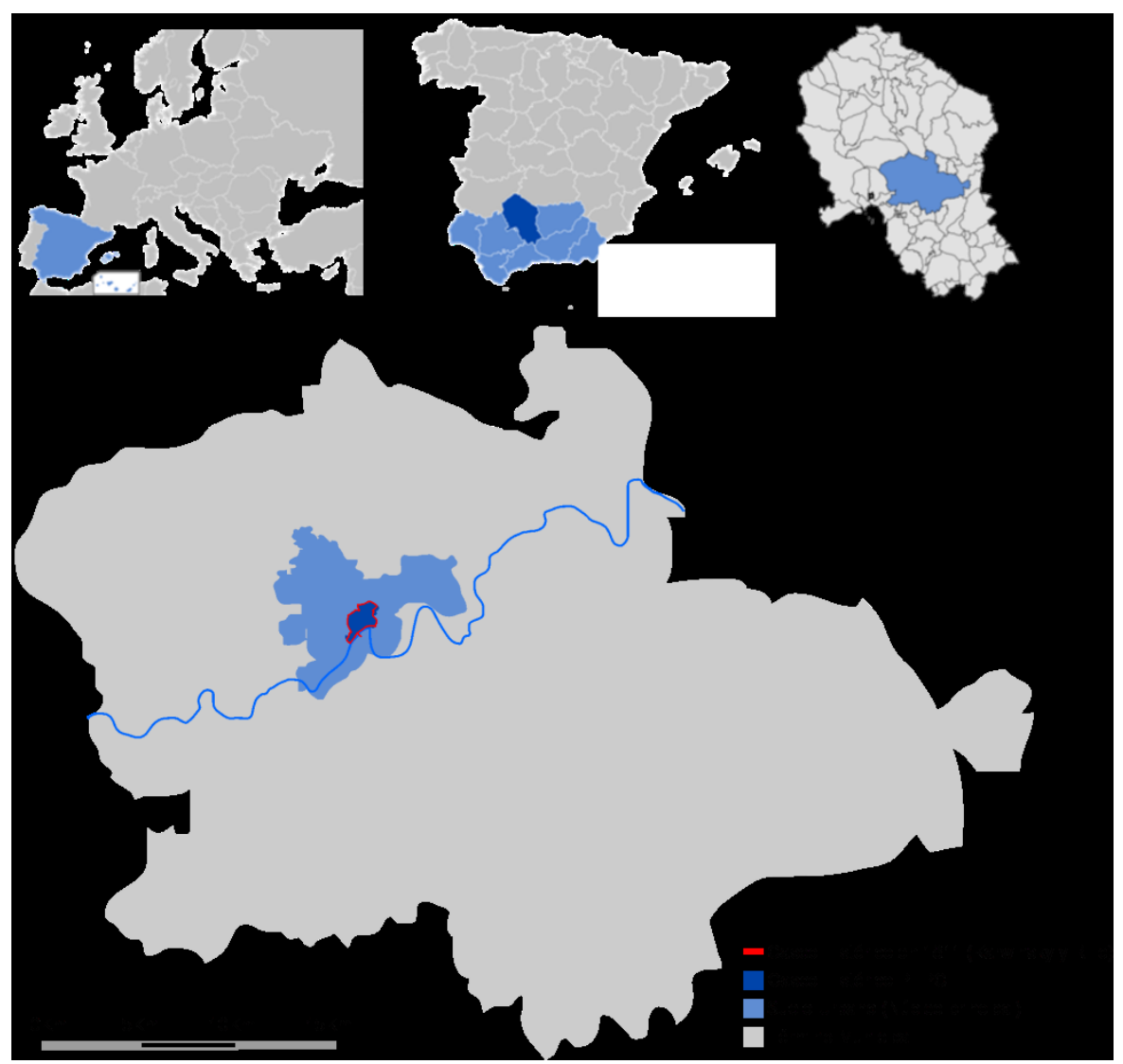

Fuente: Elaboración propia a partir del Plan General de Ordenación Urbana (PGOU), Plan Especial de Protección del Casco Histórico (PEPCH) y el primer plano conocido de la ciudad, encargado por el Ayuntamiento de Córdoba a Karwinsky y Rillo en 1811, conocido como "Plano de los franceses".

Así, y a pesar de que las bases del concurso mantienen de forma invariable el área donde deben estar ubicados los patios para participar en el CPP, el número de patios de "arquitectura antigua" han ido decreciendo paulatinamente y aumentando los de arquitectura moderna o renovada ${ }^{2}$. Dentro de esta tendencia se encuentra igualmente el detrimento de patios de casas multifamiliares, donde tienen lugar la mayor parte de las actividades sociales que reconoce y premia el concurso:solidaridad, vida en común, participación colectiva en el cuidado y arreglo del patio, así como integración del patio en la vida cotidiana ${ }^{3}$. En el año 2004 se confirma la inversión en el tipo de patios que solicitan su inscripción en el CPP (Fig. 2) y es cuando el ayuntamiento decide por primera vez la realización de un expediente que permita a los patios de Córdoba optar a su reconocimiento como Patrimonio Inmaterial de la UNESCO como medida urgente de salvaguardia.

2 Ver fig. 1. Plano de Situación. El "centro histórico" de la ciudad de Córdoba incluye la Villa o Medina y la Axerquía. La primera está conformada por la Judería y el barrio de San Basilio, o Alcázar viejo; la segunda, engloba los barrios de Santiago, Santa Marina, San Pedro, San Andrés, San Lorenzo y La Magdalena.

3 Según consta en el Informe de la Comisión Técnica del Concurso de Patios del año 2002. Consultado en el Archivo Municipal del Ayuntamiento de Córdoba. 
Figura 2 - Serie histórica de inscripciones en Concurso de Patios Populares (2003-2013)

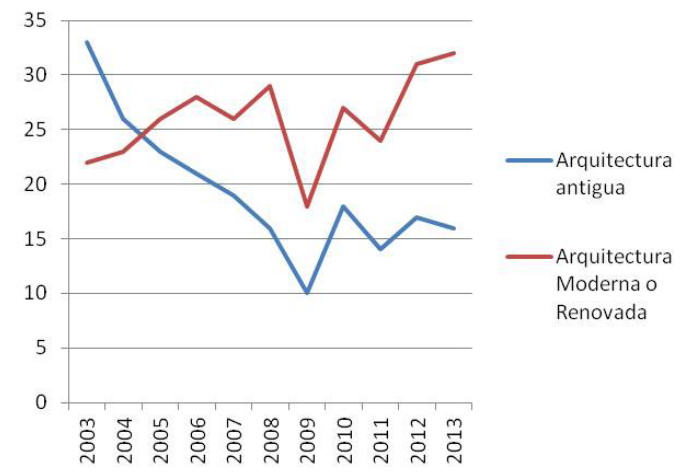

Fuente: Elaboración propia a partir de las actas del jurado del concurso en el periodo estudiado. Consultadas en Archivo Municipal del Ayuntamiento de Córdoba.

El objeto de este artículo es ofrecer argumentos para que la interpretación de los patios de Córdoba como "paisajes interiores" y como lugares de "emoción y memoria", nos permita definir nuevas categorías que atiendan precisamente a todo aquellos valores "intangibles" que las personas que habitan los patios aportan para que la FPC haya llegado a ser considerada "patrimonio de la humanidad".

\section{Estado de la cuestión: el "patio" en Córdoba como objeto de estudio}

\subsection{Revisión bibliográfica}

Al abordar la revisión bibliográfica de los trabajos de investigación que han tenido como objeto principal de su estudio el "patio" en Córdoba, o bien el espacio ya sea doméstico o urbano, que lo acoge, encontramos dos hitos fundamentales: el "I Encuentro de Académicos en Córdoba", que tuvo como tema "El patio cordobés en la historia y en la cultura andaluza", dirigido por Peláez en 1991 y cuyas conferencias fueron publicadas en el no 121 del Boletín de la Real Academia de Córdoba de Ciencias, Bellas Letras y Nobles Artes en 1992; y, apenas seis años después, el congreso "Córdoba en la Historia: la Construcción de la Urbe", cuyas actas fueron publicadas por el Ayuntamiento de Córdoba y la Fundación La Caixa en 1999.

La primera publicación incluye revisiones sobre los patios como fuente de inspiración poética (Salcedo, 1992: 279); su aparición en la narrativa española (Ocaña Vergara: 297); o bien su uso como recurso escénico (Cruz, 1992: 319). Además, se dedicaron conferencias a la figuración de los patios en la pintura de Rafael Botí (Aroca, 1992: 329) o el empleo de su luz y color como inspiración en la poesía, la narrativa o la pintura (Ojeda, 1992: 291). También se abordaron el derecho y la regulación jurídica del uso, tanto del patio en sí como de los elementos comunitarios que éste acoge, en las casas de vecinos (Palacios, 1992: 287) y una recopilación histórica de la normativa que ha afectado al centro histórico 
de la ciudad, desde los inicios del siglo XIX hasta los años 80 del siglo XX (Mir, 1992: 309). De cuyas referencias bibliográficas resaltamos los trabajos de Ramírez de las Casas Deza (1856) y Torres Balbás (1950) (citados en Lope, 1992: 317). Finalmente, el trabajo presentado por el coordinador de las jornadas, da cuenta de los "patios" como patrimonio cultural de Córdoba, incidiendo en aspectos tanto históricos, como sociológicos, botánicos e incluso, de nuevo, poéticos (Peláez, 1992: 341). Sus referencias bibliográficas principales son los "Paseos por Córdoba" de Ramírez de Arellano (1873), y los trabajos de Escobar sobre vida urbana y vivienda cordobesas en la Baja Edad Media $(1985,1989,1990)$.

En general, la mayor parte de lo reseñado hasta aquí sobre los patios de Córdoba resulta ser un "canto lírico", a la manera empleada por Gracia Boix en su pregón o exorno realizado en 1977 a propósito de la creación de la "Asociación de Amigos de los Patios Cordobeses", y del que la publicación de 1992 también da cuenta (Gracia, 1992: 337). Las emociones que despiertan, los recuerdos de infancia y la nostalgia, el sentido de pertenencia y la explosión de los sentidos. Son algunas de las constantes que aparecen en tanto en testimonios como en trabajos de los académicos cordobeses. Sus contribuciones son importantes para comprender los "patios", pero cobran real interés científico cuando es posible su comparación con los trabajos presentados en el congreso "Córdoba en la Historia: la Construcción de la Urbe". Estas actas incorporan trabajos que importan a esta investigación desde la arqueología (Murillo et al., 1999: 129; Carrillo, 1999: 75), la historia (Hernández, 1999: 271; Gómez, 1999: 345) y la geografía (García, 1999: 407).

Las aportaciones sobre el contenido botánico de los "patios" se han abordado como "ornamentación vegetal" desde una perspectiva arquitectónica (Escribano, 1956), o bien dentro de una obra más generalista que identifica y localiza geográficamente las especies vegetales empleadas en los patios andaluces (Salinas, 1985). Desde los estudios ambientales se aporta un trabajo comparativo entre las ciudades europeas de Leipzig-Halle y Córdoba y el rol que los espacios verdes desempeñan para la salud y la calidad de vida de sus habitantes (Priego et al., 2012; Priego, 2011). Otro trabajo aborda la razón de ser del "patio" a partir de la "ontología del jardín" propuesta por el filósofo italiano Rosario Assunto (Colmenarejo, 2010).

En 2009 se publicaron las conferencias que conformaron el ciclo La Fundación de la ciudad de Córdoba y su evolución histórica. Contribuyen a la profundización de esta investigación las aportaciones de Rodríguez sobre la Corduba romana, en particular sus notas sobre el origen de la ciudad (Rodríguez, 2009: 23-34); las realizadas por Escobar sobre los orígenes de la ciudad cristiana en la época Bajomedieval, en concreto sobre la organización y estructura urbana de la ciudad y características y tipologías de viviendas (Escobar, 2009: 88-98, 109-113); y las de López Ontiveros respecto a la imagen de los patios de Córdoba reflejada en la literatura viajera en los siglos XVIII y XIX (López, 2009: 172-177). Sobre este último asunto es reseñable igualmente el trabajo realizado por Baltanás sobre el ciclo andaluz en la literatura de viajes en los siglos XIX y XX, concretamente recoge testimonios sobre los patios andaluces procedentes de escritos de Amicis, Clarín, los Quintero o Amós de Escalante (Baltanás, 2003: 77, 80, 246-248). 


\subsection{El "patio" como objeto de estudio. Aportaciones a esta investigación}

García Entero (2003) muestra en su investigación sobre los jardines de las villas de Hispania cómo las domus de las clases dirigentes romanas se caracterizaron por contar con una serie de amplios espacios vacíos, más que con una sucesión de salas cerradas. El patio cubierto, claustro o pórtico o el jardín con juegos de agua son la fuente principal de iluminación y aireación de la vivienda. La vida en las domus acontece en los vacíos centrales, mientras las habitaciones son pequeñas y apenas se utilizan para dormir o leer (Thébert, 1989: 308). El peristilo, de origen griego, tiene un papel esencial no sólo en la composición arquitectónica sino en la organización general de la casa. Su incorporación al programa edificatorio de las domus romanas, que ya contaban con atrium y hortus, supone además incorporar el espacio abierto, hasta entonces sólo presente en el espacio público, como espacio de representación de poder. Efectivamente, contar con este "espacio inútil" dentro de la vivienda es un lujo que muy pocos pueden permitirse (García, 2003:56) entre otras razones porque está regulado de forma muy estricta: sólo ciudadanos nobles pueden contar con estos "regios" elementos (Vitrubio VI, 5 y VI, 3, 5, citado en García, 2003). La villa romana de "El Ruedo" en Almedinilla (Córdoba) es considerado un ejemplo paradigmático de la importancia que llegó a tener el jardín-peristilo en la domus romana como lugar de representación, en particular para la celebración del convivium.

Como han mostrado Murillo et al. (1999) la Qurtuba andalusí (711-1236), se articula de extremo a extremo gracias a las calles principales que parten de las siete puertas de la ciudad, atendiendo en buena parte al viejo cardo máximo de la Colonia Patricia. Del mismo modo, el espacio doméstico musulmán sigue un proceso de continuidad a partir del caserío romano, en el que el patio, como lo fue el peristilo, constituye el elemento articulador principal de la vivienda andalusí, al menos en la Madina. Se señalan sin embargo dos diferencias notables: en primer lugar, la casa islámica tiene un carácter introvertido: es diseñada desde adentro hacia afuera y emplea, además, una arquitectura más flexible, cuyas modificaciones serán siempre absorbidas a través del espacio libre interior. La casa andalusí cuenta con una sola puerta, a la que da servidumbre un adarve, callejón sin salida que penetra en el corazón de las manzanas edificadas, más que una calle, lo que refuerza este carácter. Hacia adentro, la puerta conduce a un zaguán, y éste directamente al patio, el corazón de la casa, al que se abren todas las habitaciones (Murillo et al., 1999: 131-150).

Hernández (1999) ha demostrado que a fines de la Edad Media las "casas de vecinos" constituyen una tipología de vivienda muy extendida en Córdoba. Las también denominadas "casas de vecindad", "casas de partido" o "patios de vecindad", son resultado del aprovechamiento de otras edificaciones - en especial conventos y casas señoriales- que, al compartimentarse, pudieron albergar a distintas familias en alojamientos y viviendas reducidas (Hernández, 1999: 271). Este carácter de "viviendas de uso compartido" se encuentra también en los "corrales" o "casas corrales", una estructura típica del siglo XVI que responde sin embargo a un programa arquitectónico concebido como tal. Se trata de una crujía perimetral en galerías de dos o más plantas de altura, que vuelca hacia 
un espacio interior o patio (Morales, 1981: 14). Ambas formas constituyen una unidad urbanística que se caracteriza por la yuxtaposición de espacios de uso privado y común, siendo los servicios comunes más habituales la cocina, la pila, el pozo, la casa-puerta, el corral y el patín o patio (Hernández, 1999: 274). Las conclusiones que interesan a nuestra investigación son dos: en primer lugar que las viviendas en las que los elementos y servicios comunes superan a los espacios privados son las más numerosas, representando un 58,2\% del total (Hernández, 1999: 280); además, que la mayor parte de estas casas se ubicaban en los barrios más populosos de la ciudad en los siglos bajomedievales: San Pedro, San Andrés, Santa Marina y Santa María (Escobar, 1989: 186). Es en estos barrios, que conforman la zona conocida como Axerquía, dónde han pervivido las casas de vecinos, muchas de ellas aún en uso y habituales en el CPP. (Fig. 3)

Figura 3 - Plano Situación de Casas-patio de vecinos de Córdoba
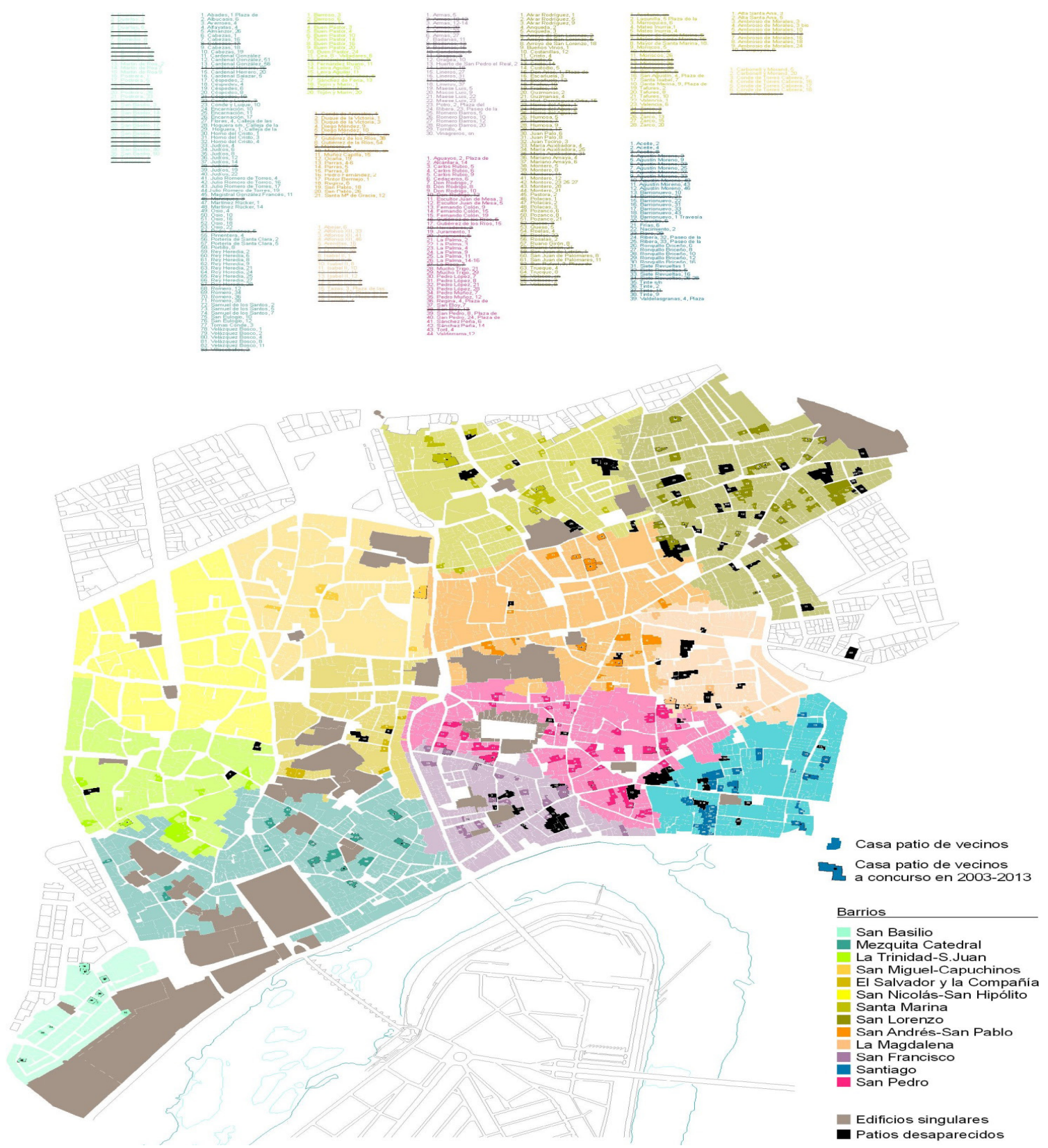

Fuente: Elaboración propia a partir de plano original de Esteban y Obrero (2012) y actas del jurado del Concurso Municipal de Patios Populares (2003-2013) 
Los estudios sobre las dos unidades urbanísticas fundamentales: Villa y Axerquía, que conformaban la ciudad de Córdoba tras la reconquista en el año 1236, y que sin grandes cambios llegan hasta bien entrado el siglo XIX, pueden encontrarse en los trabajos de Escobar $(1999,2009)$. Así, se distinguen la Villa, antes Madina, con una clara ascendencia musulmana; la Axerquía, que el autor denomina urbanismo mixto islamocristiano (Escobar 1999: 198), incorporándose en el siglo XV el denominado Alcázar Viejo, donde se llevó a cabo un urbanismo medieval tardío, geométrico y muy bien conservado, totalmente diferenciado del islámico de la Villa (Escobar, 2009: 105). Son estas tres áreas las que conforman el área de influencia del CPP actualmente, y por tanto de la FPC, como ya se ha visto aquí. Los topónimos de estas catorce circunscripciones iniciales, también denominadas collaciones, desiguales en extensión, coincidían con las respectivas advocaciones religiosas de sus parroquias ${ }^{4}$. Esta división administrativa dará lugar a los barrios ya en época moderna, que han mantenido generalmente estos nombres hasta la actualidad. (Ramírez, 1948: 22-23)

\section{Dos propuestas para una nueva interpretación de los patios}

El objeto de interés en la gestión del patrimonio ha evolucionado a partir de conceptos como "riquezas", "tesoros" o "antigüedades", susceptibles siempre de conservarse en un museo como colecciones representativas de determinadas civilizaciones, hacia su comprensión como expresiones culturales, en las que el contexto comenzó a tener un papel determinante especialmente a partir de la Convención de Patrimonio Mundial de la UNESCO, firmada originalmente por 183 países, en 1960. El "bien cultural" fue así ampliando su área de interés hacia manifestaciones de la actividad humana en general, como folklore, fiestas tradicionales o documentos privados, aunque históricamente sean relativamente recientes (Prats, 1997). Actualmente, el patrimonio cultural es considerado, además de legado y soporte de la identidad colectiva, como un recurso fundamental de desarrollo. Su aprovechamiento como recurso turístico es considerado un riesgo para su conservación, pero también una oportunidad para el desarrollo humano y económico de las ciudades históricas (de la Calle et al., 1998). En la década de los cincuenta, Freeman Tilden aportó la filosofia que subyace en el oficio de quienes se ocupan de "comunicar" el patrimonio. La "interpretación del patrimonio" nació así para revelar los valores no evidentes que se encuentran en áreas naturales protegidas (Tilden, 2006), pero se extendió rápidamente como un recurso educativo y de comunicación aplicable al resto de bienes patrimoniales, fuesen estos tangibles o intangibles.

4 Siete de estas collaciones pertenecían a la Villa: Santa María, San Juan, Omnium Sanctorum, San Nicolás, San Miguel, San Salvador y Santo Domingo; y otras siete a la Axerquía: San Nicolás, San Pedro, San Andrés, Santa Marina, San Lorenzo, Santa María Magdalena y Santiago. En el siglo XIV se incorpora la collación de San Bartolomé, no adscrita a ninguna parroquia, y que comprendía la antigua Judería y el AlcázarViejo. 
En el año 2000, la Asociación Nacional para la Interpretación del Patrimonio de Estados Unidos definió su actividad como un proceso de comunicación que produce conexiones emocionales y cognitivas entre los intereses del público y los significados inherentes al recurso.

El modelo más reciente de "patrimonio" busca sostener la vida de una tradición mediante el reconocimiento de las condiciones necesarias para la reproducción cultural. La tarea consiste por tanto en mantener el sistema en su conjunto como una entidad viva, lo que implica superar la idea de conservación centrada en los "tangibles", o "bienes inmuebles". Ello implica que el valor de dicha tradición debe acordarse entre sus "portadores" y "transmisores", y que deben tenerse en cuenta tanto sus hábitos de vida (habitus) como el espacio en el que se desarrolla la vida social (habitat) (KirshenblattGimblett, 2004). El patrimonio inmaterial está por tanto íntimamente ligado a la identidad de un pueblo o una población a través de su memoria y sus emociones, y éstas a su vez profundamente ancladas en la historia a través de lo representado como tangible.

\subsection{Los Patios de Córdoba como "Paisajes interiores"}

El Convenio Europeo del Paisaje, redactado como "Carta de Florencia" en el año 2000 y ratificado por España en 2008, define "paisaje" como cualquier parte del territorio tal como la percibe la población, cuyo carácter sea el resultado de la acción y la interacción de factores naturales y/o humanos; considerando además que el "paisaje" es un bien público, objeto de derecho de las poblaciones que lo perciben y para cuyo disfrute es preciso generar actitudes no solo de protección, sino también de gestión y ordenación (Consejo de Europa, 2000).

El panóptico de la ermita de la Virgen de la Sierra en Cabra fue reconocido como el "punto de vista más abarcador de toda Andalucía", y como tal fue declarado Sitio de Interés Nacional en 1929 (López Ontiveros, 2002). El paisaje es allí percibido desde un punto de vista comprensivo, un tour d'horizon, que da la impresión de existir "en un vacío". Frente a este, los paisajes interiores quedan "poetizados" como proyección de un estado de ánimo (Clarke, 1977), recogidos, contenidos en una composición arquitectónica de la que son hueco fundamental. Nos serviremos aquí del concepto "vacío" como referencia para definir ambos: mientras en los primeros el vacío es contenedor, derredor o soporte; en los segundos, el vacío es contenido, tejido o lugar.

En el centro histórico de Córdoba, prácticamente cada casa cuenta con su propio patio y, a su vez, las casas de vecinos cuentan con uno o dos patios comunes. Tamaño y forma varían, las orientaciones también. Permanece el hueco, el vacío que penetra la imagen compacta de una ciudad que se aferra a la alineación de sus edificios y deja mínimo espacio a las calles. El espacio se gana hacia dentro, en los patios. Un paisaje que es un espacio a cielo abierto pero que también se deja entrever a través de las cancelas cerradas pero transparentes que permiten comprender el espacio doméstico también, de algún modo, como público en tanto que se comparte visualmente con todo el que pasa junto a la puerta. 
El paseo, el deambular por la ciudad, también puede sernos útil para definir este tipo de paisajes. Así, es mediante el movimiento consciente o inconsciente por el espacio público, por sus calles, callejas, adarves y plazuelas, lo que nos permite conectar y relacionar mediante itinerarios los espacios privados, cerrados y estáticos que conforman los paisajes interiores. Los vacíos del espacio público son aquí contenedores que se articulan como hilo conductor hacia lo cotidiano, íntimo y permanente que subyace en los vacíos como contenidos, que representan los patios, retazos de vida, universos sumergidos, listos para ser descifrados. La ciudad es el líquido amniótico en el cual todo crece y se transforma a través de los sentidos de quien pasea y encuentra (Careri, 2002: 86), el patio es el hallazgo, el objeto vivido, un paisaje interior de permanencia en constante transformación gracias a la emoción y la memoria de quienes lo habitan, pero también de quienes lo descubren felizmente:

Sus casas blancas con rejas verdes y en el centro de las casas construidas en cuadrado, estos patios rodeados a veces de galerías en arcadas. Este es el salón, el parterre, el paraíso terrestre, pues en comparación no valen nada las habitaciones más suntuosas de los palacios del Norte. Los plataneros de hojas inmensas, los naranjos, los limoneros, los jardines los llenan con sus suaves emanaciones y los decoran con sus flores y frutos; ellos expanden su frescor delicioso, aumentando frecuentemente por el murmullo inagotable de una fuente. (...) En medios de los ardores del verano, cuando el turista camina con dificultad tras la búsqueda de vestigios romanos y de ruinas moras, su frente sudorosa recibe al pasar por el umbral de esas moradas encantadoras los efluvios olorosos y refrescantes de los patios misteriosos. Era para nosotros una tentación - a costa de una violación de domicilio - penetrar en ellas para gozar de su frescor. (Godard, 1862: 195-196; Citado en López Ontiveros, 2009: 173)

Podríamos concluir que lo que define el patio como "paisaje interior", además de su razón inherente de vacío interior en la ciudad y de lo construido, son una serie de elementos que incluyen: a) su localización "geográfica", que aporta una identificación con un barrio, pero también el tipo de calle o plaza en el que se ubica la casa que contiene el patio; b) elementos que "identifican" el patio con la tradición, en el sentido de continuidad, desde una perspectiva arquitectónica, botánica, de composición, y de formas de mantenimiento; c) elementos que "identifican" el patio con la innovación, en el sentido de regeneración de la tradición, de nuevo desde una perspectiva arquitectónica, botánica, de composición, y de formas de mantenimiento; d) elementos "inmateriales" como el silencio, los sonidos asociados al agua o a la presencia de fauna, aromas, o colores, que identifican el patio.

\subsection{Los Patios de Córdoba como lugares de emoción y memoria}

En este epígrafe nos ocuparemos primero de definir qué entendemos por "lugar", y para ello trataremos de responder a las cuestiones: ¿qué es habitar? y ¿dónde reside la 
esencia del habitar? Al abordar la primera cuestión atenderemos la teoría del "genio del lugar" (Genius loci), tan querida para la arquitectura y el paisajismo modernos. Como ha estudiado García Remón (1997) el genius loci supone por un lado una conceptualización tomada de la Antigüedad Clásica, en la que los símbolos rememoran aquello mítico del lugar, previo a la intervención del ser humano y que éste en tanto que creadorconstructor debe saber interpretar; y por otro, el concepto se reinventa en el siglo XVIII como metáfora central de la nueva poesía inglesa ilustrada en la que el genius loci se identifica como la esencialidad canónica del paisaje, entendiendo éste como recipiente de la historia, la religión y el espíritu del país. El jardín deja entonces de interpretarse pasivamente desde su planta y es concebido para una experiencia individual, su pretensión última es ser "reconocido" mediante el paseo y no antes (García-Remón, 1997:203-204). El lugar es, en ambos casos, un espacio existencial que revela nuestro estar en el mundo como sujetos, una idea que Heidegger expresaría en la conferencia "Construir, habitar, pensar" (1951) sirviéndose de las conexiones lingüísticas de los términos en alemán, "El modo como tú eres ( $d u$ bist), yo soy (ich bin), la manera según la cual los seres humanos somos en la tierra es el buan, el habitar." (Heidegger, 1994:142) Hay algo más que define el habitar y con ello el lugar, construir como habitar implica en primer lugar el pensar, y además el cuidar de quien habita lo construido.

El cuidar, en sí mismo, no consiste únicamente en no hacerle nada a lo cuidado. El verdadero cuidar es algo positivo, y acontece cuando de antemano dejamos a algo en su esencia, cuando propiamente re-albergamos algo en su esencia; cuando, en correspondencia con la palabra, lo rodeamos de una protección, lo ponemos a buen recaudo. El rasgo fundamental del habitar es este cuidar (mirar por). (Heidegger, 1994: 144)

La vivienda, el lugar doméstico, es un ámbito fundamental para comprender lo que la infancia, la vida familiar o la muerte, representan en la historia de la humanidad. No en vano, el término domus engloba tanto el marco arquitectónico como a quienes lo habitan, por ello se ha considerado que la idea de domus reviste una dimensión religiosa, social y económica (Thébert, 1989: 399). Del mismo modo que domus significa más que estructura arquitectónica, se ha considerado que el espacio doméstico andalusí refleja la historia, el crecimiento acumulado y la estructura familiar de varias generaciones (Murillo et al. 1999: 144). En ambos casos, podemos considerarlos como espacios de memoria fuertemente vinculados a sus habitantes y sus formas de vida. Los objetos que están arquitectónicamente fijados a un lugar a través de la memoria incluyen ideas y sentimientos, que son así entendidas como fundamentos de la composición. Carruthers explicó en su estudio sobre la relación entre memoria y lugar, que "un recuerdo-imagen es afectivo por naturaleza, es decir, necesariamente derivado de los sentidos y, por tanto, cargado de emociones. El recuerdo es una recreación de la experiencia, lo que implica imaginación y emoción" (Citado en Bruno, 2002: 222).

La propuesta de Heidegger podría ser un "somos porque habitamos (el lugar)", mientras la de Bruno es un "somos porque sentimos (el lugar)", distinguiéndose aunque 
no frente a ellas la teoría del genio del lugar vendría a afirmar que "un lugar es porque es percibido". Habitamos por tanto aquellos lugares que sentimos, que están ligados a nuestras emociones a través de la experiencia y la memoria.

Durante la época de sobre-modernidad nos hemos dado, sin embargo, lugares de inmemoria.Augé (2006) mantiene que proliferan los espacios que no se viven ya ordenados en torno a un campanario, la sirena de un colegio, $u$ otro ritual que marque el paso del tiempo, que delimite nuestra existencia en una temporalidad espacial ${ }^{5}$. Los aeropuertos, las áreas de servicio de las autopistas, los hospitales, los hoteles, y también los campos de refugiados, las barracas de miseria, son todos ellos "espacios" pero no "lugares", espacios construidos que han perdido su conexión con el habitar y el cuidar, por seguir el discurso de Heidegger. Un lugar implica, para Augé, la posibilidad de un recorrido, de un discurso, de un lenguaje que lo caracterice, una "práctica del espacio" que permite "repetir la experiencia alegre y silenciosa de la infancia; ser otro y pasar al otro" (Certeau, citado en Augé, 2006: 89), que se opone diametralmente a aquellos espacios donde el individuo se siente como espectador sin que la naturaleza del espectáculo le importe verdaderamente.

El espacio del no-lugar no crea, en definitiva, ni identidad singular ni relación, sino soledad y similitud. Tampoco da lugar a la historia, o a su relación con la emoción o la memoria, sino que transforma éstas, eventualmente, en elementos del espectáculo. ¿Dónde reside entonces la esencia del habitar? A partir de lo expuesto creemos más fácil argumentar que reside en las emociones y en la memoria de quienes habitan. Nos apoyaremos primero en Nussbaum $(2008,2013)$ quien argumenta que una correcta valoración y cultivo de las emociones es fundamental para la vida social y democrática (Nussbaum, 2013:3-9) y quien ya estableció que las emociones son una forma de juicio valorativo, que conforman por tanto el pensamiento racional, que atribuye a ciertas cosas y personas fuera del control del ser humano una gran importancia para el bienestar y el florecimiento propios. Las emociones tienen "un objeto hacia el que dirigirse y por el que dirimirse" (Nussbaum, 2008: 53) y es el papel que desempeña dicho objeto para la propia vida del sujeto lo que sustenta el valor de dichas emociones. Las emociones tienen además una historia, y no es posible comprenderlas en muchos casos si no es como parte de una experiencia personal en el tiempo, aunque ellas mismas sean una construcción social (Nussbaum, 2008: 176-179-206).

Basándonos en la revisión bibliográfica realizada, es posible concluir que las emociones y la memoria de la comunidad que habita y cuida los patios en Córdoba están basadas en aquellos elementos tradicionales que conforman el "patio" de Córdoba como lugar, y que Peláez sintetizó en 1991:

"Para concluir, conviene reducir a siete los elementos que configuran al
patio popular. En primer lugar, las flores: geranios, claveles, fucsia y begonias,
rosas, jazmines y damas de noches, helechos y albahaca, naranjos, limoneros
y palmeras. En segundo lugar, el agua de la fuente del pozo, del botijo o del tiempo perdido, Vol I ("Por el camino de Swann"), capít. 5-6, Madrid, Alianza. 
del cántaro, para verterla en las macetas, en el empedrado o en el ambiente, como refresco o como alimento. En tercer lugar, la maceta o tinaja, como elemento de recepción de la flor o de la vegetación. En cuarto lugar, la cal ó pintura, como color de fondo del espacio que configura el propio patio. En quinto lugar, aunque no necesariamente, la columna o el arco. En sexto, el guijarro.Y presidiéndolo todo, principio o final, el elemento humano, mujer u hombre, que dedica sus afanes al cuidado y esmero del lugar." (Peláez, 1991:349)

La vivencia de la FPC cumple, además, con las características que Paloma Aguilar asimila al concepto de "memoria colectiva" ya que contiene tanto los recuerdos que la comunidad tiene de su propia historia, como los conocimientos aprendidos y transmitidos, extraídos como valores a través de la vivencia de dicha historia (Aguilar, 1996). Como la memoria colectiva suele fijarse en las instituciones y se revive periódicamente mediante ceremonias y ritos públicos, ésta forma una suerte de patrimonio común con el que la persona se encuentra desde que nace y que se imbrica con sus propios recuerdos individuales (García-Álvarez, 2009). De este modo, mediante la afirmación del tiempo, el espacio y de los lugares comunes, se pretende una memoria activa, encarnada en la comunidad, que conforma su espacio de vida a través de elementos simbólicos que forman un continuum entre lo tangible y lo intangible.

Siguiendo esta argumentación, es posible también afirmar que los patios de Córdoba son "espacios de supervivencia de memoria", según un concepto que se define como "aquellos lugares y grupos que, dentro de un país, de una región o de una ciudad, conservan vivas las tradiciones, valores y formas de vida propias del pasado y al margen de los espacios controlados oficialmente" (Legg, 2005). Los patios que participan en el concurso, y que quedan de este modo "institucionalizados", son una pequeña parte (48 en la última convocatoria) del total de patios que podrían considerarse interesantes desde el punto de vista patrimonial, unos 276 según un último censo realizado por Esteban y Obrero, ya mencionado.

El concepto "lugar de memoria" es tan potente que ha sido empleado por diferentes culturas como fórmula para construir la "memoria pública", una memoria que es ajena a los grandes monumentos o patrimoniales descritos y preservados "libres de emociones". Así, Hayden (1995) considera que los lugares de memoria son la clave para que los ciudadanos puedan construir su pasado de forma colectiva, al mismo tiempo que compartir esta experiencia con "visitantes", ajenos a dicha historia colectiva, puede ser una forma de darse a conocer como colectivo en el presente (Hayden, 1995: 46), lo que tendría que ver con la identidad cultural que se genera en la confluencia entre lugar, memoria y colectividad. Una confluencia que encontramos en la siguiente descripción de "lugar de memoria" realizada por Casey (1987): "Es la conservación persistente de un lugar como contenedor de experiencias lo que contribuye a su condición de lugar de memoria. Una memoria viva y alerta que conecta directa y espontáneamente con el lugar es la garantía más eficaz para conservar su identidad y autenticidad" (Casey, 1987: 186-187). 
Al incorporar las emociones y la memoria al análisis pretendemos aportar una valiosa revisión de la historia pública de la ciudad.

\section{CONCLUSIONES}

El reconocimiento de la Fiesta de los Patios de Córdoba como Patrimonio Inmaterial de la Humanidad por la UNESCO es una oportunidad para acometer un trabajo de investigación sistemático sobre el tipo de vida que ha estado, y aún está, asociada a las casas-patio, que permita avanzar en su definición, naturaleza y características. Es posible extraer dos tipos de conclusiones a partir del trabajo presentado. En primer lugar, la revisión bibliográfica nos muestra la pervivencia de las siguientes características de las viviendas con "patio" en Córdoba:

- Heredan de la domus romana sus funciones higiénicas de aireación, iluminación y suministro de agua, además de lugar para la vida cotidiana en común, mientras se mantiene la limitada superficie de dormitorios y resto de habitaciones "cerradas".

- Conservan de la casa andalusí su carácter introvertido, y de la trama urbana basada en los adarves, callejas sin salida, un paisaje urbano laberíntico que parece también mirar siempre "hacia adentro".

- Perdura en la ciudad la distinción, presente desde el siglo VIII, entre Madina (denominada Villa en el Medievo) o zona alta de la ciudad y espacio geográfico tradicional de ubicación de las casas unifamiliares de las familias más acaudaladas y Axerquía, que contiene los barrios más populares. Ambas conforman, junto al Barrio de San Basilio o Alcázar Viejo, el área de localización de los patios que pueden presentarse a concurso.

- La ciudad alberga, desde la Baja Edad Media, una mayor proporción de casas de vecinos, y por tanto de patios de uso comunitario, en la denominada Axerquía.

Unas conclusiones que se refieren esencialmente al tipo de trabajos realizados hasta la fecha sobre las casas-patio de Córdoba, estrechamente ligadas a la casa-patio como estructura y no tanto como contenedor de experiencias. El segundo tipo de conclusiones se refieren a la propuesta presentada en este trabajo, y se refieren a la necesidad de poner en evidencia la relación entre los trabajos realizados sobre arqueología, historia, o la existencia actual de elementos construidos, documentados científicamente según se ha mostrado aquí, y la memoria y emociones de quienes habitan las casas, que revelan los trabajos que abordan los patios de Córdoba de forma específica. En este sentido, es reseñable en primer lugar, que si se ha conseguido mantener de forma casi invariable la vitalidad del CPP que origina la FPC, y con ello el reconocimiento de la UNESCO, ha sido porque se han activado, promovido y cuidado elementos como la transmisión de conocimiento de forma sincrónica, entre vecinos y cuidadores, como diacrónico, entre diferentes generaciones abuelos-abuelas y nietos-nietas. $\mathrm{O}$ bien entre padres-madres e 
hijos-hijas; la elevada valoración entre vecinos y cuidadores de las actividades realizadas en comunidad, tanto lúdicas y festivas como de trabajo activo para conseguir abrir el "patio" para el CPP; la elevada integración del "patio" y de su cuidado y mantenimiento en la vida cotidiana de la comunidad; y todo ello eludiendo los riesgos de mercantilización evidentes a los que se ve sometida la fiesta. Se ha intentado mostrar aquí que estos valores residen precisamente en la memoria, individual y colectiva, y en las emociones de quienes habitan y cuidan los patios. La relación entre el lugar, "patio", y memoria colectiva ha permitido además generar una fiesta con identidad propia, que ha eludido los estándares de homogeneización cultural. Las casas-patio han aportado además a la memoria colectiva de la ciudad el punto de anclaje espacio-temporal necesario para conservar una forma de vida reconocible como una seña de identidad comunitaria.

\section{BIBLIOGRAFÍA}

AGUILAR, Paloma. Aproximaciones teóricas y analíticas al concepto de memoria histórica. Madrid: Instituto Universitario José Ortega y Gasset, 1996.

AROCA,Ángel. El patio cordobés en la pintura de Rafael Botí. Boletín de La Real Academia de Córdoba, n. 121 , p. 329-332, 1992.

AUGÉ, Marc. Los “No Lugares” espacios del anonimato. Una antropología de la sobremodernidad. Barcelona: Gedisa, 2006.

BALTANÁS, Enrique. La materia de Andalucía: el ciclo andaluz en las letras de lossiglos XIX y XX. Sevilla: Fundación José Manuel Lara, 2003.

BRUNO, Giuliana. Atlas of Emotion: Journeys in Art, Architecture, and Film. London:Verso, 2002.

CARERI, Francesco. Walkscapes. El andar como práctica estética. Land\&Scape Series 1. Barcelona: Gustavo Gili, 2002.

CASEY, Edward. Remembering: A Phenomenological Study. Bloomington: Indiana University Press, 1987.

COLMENAREJO, Rosa. Aproximación a una ontología del patio. Paisajes cotidianos para comprender el siglo XXI. Alfa, revista de la asociación andaluza de filosofía, XIII, 26-27, p. 359-368, 2010.

Consejo de Europa. “Convenio Europeo Del Paisaje”. ETS nº176. Florencia: Consejo de Europa, 2000.

CRUZ, Antonio. “El patio como espacio escénico.” Boletín de La Real Academia de Córdoba, n. 121, p. 319-327, 1992.

DE LA CALLE, Manuel, y GAR CÍA, María. Ciudades históricas: Patrimonio cultural y recurso turístico.” Ería. Revista Cuatrimestral de Geografía, n. 47, p. 249-266, 1998.

DE LOPE, José Luis. Evolución histórica-arquitectónica del patio cordobés. Boletín de La Real Academia de Córdoba, n. 121, p. 313-317, 1992.

ESCOBAR, José Manuel. La vida urbana cordobesa: el Potro y su entorno en la Baja Edad Media. Córdoba: Caja Provincial de Ahorros, 1985

- Córdoba en la Baja Edad Media: evolución urbana de la ciudad. Córdoba: Caja Provincial de Ahorros, 1989.

—. La vivienda cordobesa a fines de la Baja Edad Media. Baena: Asociación de Profesores de Geografia e Historia de Bachillerato de Andalucía, p. 175-183, 1990.

- La Ciudad de Córdoba Tras La Reconquista. En Córdoba en la historia: la construcción de la urbe. Actas del Congreso. Córdoba 20-23 Mayo, 1997, Córdoba: Ayuntamiento de Córdoba y Fundación La Caixa, p. 183-200, 1999. 
. La Córdoba Bajomedieval (siglos XIII-XV). El Origen de La Ciudad Cristiana. En La Ciudad de Córdoba: Origen, Consolidación e Imagen, Córdoba: Servicio de publicaciones de la Universidad de Córdoba, p. 83-127, 2009. ESCRIBANO, Víctor. Ornamentación vegetal de los patios cordobeses. Madrid: Escuela Técnica Superior de Arquitectura. 1956.

GARCÍA, Jacobo. Lugares, paisajes y políticas de memoria. Una lectura geográfica. Boletín de Geógrafos Españoles, n. 51, p. 175-180, 2009

GARCÍA,Virginia. Algunos apuntes sobre el jardín doméstico en Hispania. Anales de Prehistoria y Arqueología, n. 19-20, p. 55-70, 2003.

GAR CÍA MOLINA, Juan Antonio. La Casa tradicional cordobesa en la Edad Contemporánea. Breve aproximación a sus Características Tipológico-Constructivas. En Córdoba en la historia: la construcción de la urbe. Actas del Congreso. Córdoba 20-23 Mayo, 1997, Córdoba: Ayuntamiento de Córdoba y Fundación La Caixa, p. 407-420, 1999.

GRACIA, Rafael. El patio cordobés como lugar de encuentro. Boletín de La Real Academia de Córdoba, n. 121, p. 337-339, 1992

HAYDEN, Dolores. The Power of Place. Urban Landscapes and Public History. Cambridge, MA:The MIT press, 1995. HEIDEGGER, Martin. Construir, habitar, pensar. En Conferencias y artículos, Barcelona: Ediciones del Serbal, p. 127-142, 1994.

KIRSHENBLATT-GIMBLET, Barbara. Intangible Heritage as Metacultural Production. Museum International, n. 56 (1-2), p. 52-65, 2004.

LEGG, Stephen. Contested and Surviving Memory: Space, Nation and Nostalgia in Les Lieux de Memoire. Environment and Planning: Society and Space, n. 23 (4), p. 481-504, 2005.

LÓPEZ ONTIVEROS, Antonio. Evolución urbanística de Córdoba en los Siglos XVIIIY XIX según la literatura viajera. En La ciudad de Córdoba: origen, consolidación e imagen, Córdoba: Servicio de publicaciones de la Universidad de Córdoba, p. 133-208, 2009.

MIR, Rafael. Normativa del patio principal en la zona histórico-artística de Córdoba. Boletín de La Real Academia de Córdoba, n. 121, p. 309-311, 1992.

MORALES, Francisco. Los Corrales. Sevilla: Grupo Andaluz de Ediciones, 1981.

MURILlO, Juan Francisco, FUERTES, María del Camino, y LUNA, Dolores. Aproximación al análisis de los espacios domésticos en la Córdoba Andalusí. En Córdoba En La Historia: La Construcción de La Urbe. Actas Del Congreso. Córdoba 20-23 Mayo, 1997, Córdoba: Ayuntamiento de Córdoba y Fundación La Caixa, p. 129-154, 1999.

NUSSBAUM, Martha C. Paisajes del pensamiento. La inteligencia de las emociones. Barcelona: Paidós, 2008. Press, 2013

Political Emotions. Why Love Matters for Justice. Cambridge, MA:The Belknap Press of Harvard University

OCAÑA, José María. El patio cordobés en la narrativa española. Boletín de La Real Academia de Córdoba, n. 121, p. 297-300, 1992.

OJEDA, Antonio. Luz y color de los patios cordobeses. Boletín de La Real Academia de Córdoba, n. 121, p. 291-295, 1992.

PALACIOS, Diego. Los patios y el derecho. Boletín de La Real Academia de Córdoba, n. 121, p. 287-290, 1992.

PELÁEZ DEL ROSAL, Manuel. Los patios como patrimonio cultural de Córdoba. Boletín de La Real Academia de Córdoba, n. 121, p. 341-351, 1992.

PRATS, Llorenç. Antropología y patrimonio. Barcelona: Ariel, 1997.

PRIEGO, Carlos. Naturaleza y Sociedad: el valor de los espacios verdes urbanos. Madrid: Ministerio de Medio Ambiente y Medio Rural y Marino, 2011. 
PRIEGO, Carlos, RODRÍGUEZ-MORCILLO, Luis, and BREUSTE, Jürgen. The Courtyards of Cordoba in Spain. Social Functions of Private Spaces in Historical Neighbourhoods. Laufener Spezialbeiträge, p. 67-72. 2012.

RAMÍREZ DE ARELLANO, Teodomiro. Paseos por Córdoba, o sean, apuntes para su historia. Córdoba: Rafael Arroyo, 3 vols., 1873.

RAMÍREZ DE LAS CASAS, Luis María. Indicador Cordobés, o sea, manual histórico-topográfico de la ciudad de Córdoba, Córdoba, 1856.

- Anales de la ciudad de Córdoba. Desde el Siglo XIII y año 1230 en que fue conquistada por el santo Rey D. Fernando III, hasta el de 1850. Córdoba: Real Academia de Córdoba de Ciencias, Bellas Letras y Nobles Artes, 1948.

REMÓN, Juan. La Invención del genio del lugar. En: Javier Maderuelo (ed.) El jardín como arte, vol. 3, p. 197-206. Huesca: Diputación de Huesca y Universidad de Zaragoza, 1997.

RODRÍGUEZ CARRILLO, Sacramento. El patio cordobés como impulsor del canto del poeta. Boletín de La Real Academia de Córdoba, n. 121, p. 273-278, 1992.

RODRÍGUEZ NEILA, Juan Francisco. Corduba Romana, capital de la provincia Hispania Ulterior Baética. In: La Ciudad de Córdoba: Origen, Consolidación E Imagen, p. 23-77. Córdoba: Servicio de publicaciones de la Universidad de Córdoba, 2009.

SALCEDO, Miguel. El patio en la poesía. Boletín de La Real Academia de Córdoba, n. 121, p. 279-85, 1992.

SALINAS, Lola. La flora de los patios andaluces: conocimiento para su conservación. Córdoba: Ayuntamiento de Córdoba, 1985.

THÉBERT,Yvon.Vida privada y arquitectura doméstica en el África romana. In: Philippe Ariès y Georges Duby (Ed.) Historia de La Vida Privada,Vol. I, p. 304-401, Madrid:Taurus- Alfaguara, 1989.

TILDEN, Freeman. La Interpretación de Nuestro Patrimonio. Valladolid: Asociación para la Interpretación del Patrimonio, 2006.

TORRES BALBÁS, Leopoldo. Algunos aspectos de la casa Hispano-Musulmana: almacerías, algorfas y saledizos. Al-Andalus: Revista de Las Escuelas de Estudios Árabes de Madrid y Granada XV (1), p. 179-190. 1950.

UNESCO. Convención para la salvaguardia del Patrimonio Cultural Inmaterial. Convención MISC/2003/CLT/ CH/14. París: Organización de las Naciones Unidas para la Educación, la Ciencia y la Cultura, 2003.

- "Fiesta of the Patios in Cordova" París: Organización de las Naciones Unidas para la Educación, la Ciencia y la Cultura, 2012. 


\title{
Foundations for a new Interpretation of the Patios in Cordoba: Interior Landscape, Emotions and Memory
}

\begin{abstract}
The Intergovernmental Committee for the Safeguarding of the Intangible Cultural Heritage inscribed the "Fiesta of the Patios in Cordoba" on the Representative List of the Intangible Cultural Heritage of Humanity, in 2012. Consequently, growing recognition of the immaterial heritage, considered as a vital factor in the assertion of cultural identity. This paper includes a general review on the state-of-the-art and explores new characterization categories that intends to contribute to keeping up the heritage valuation of the "patios" based on an emotional geography from its inhabitants' identity, recognition, and memory. The aim of this paper is contributing to enrich the understanding of the "patios" way-of-life beyond the existing typological variations based on the building architecture.
\end{abstract}

Keywords: Inmaterial Heritage, Fiesta of the Patios in Cordova, Interior Landscape, Emotions, Realm of memory.

\section{Proposta para uma nova interpretação dos pátios de Córdoba (Espanha): paisagem interior, emoções e memória.}

\section{Resumo}

A inclusão da "Festa dos Pátios de Córdoba" na Lista Representativa do Patrimônio Cultural Imaterial da UNESCO, em 2012, significou o reconhecimento dos valores que a mesma possui tanto para a convivência e solidariedade humanas, como para o patrimônio natural e cultural da cidade. Este artigo inclui uma revisão bibliográfica completa sobre o estado desta questão, e propõe novas categorias tipológicas que pretendem contribuir para uma atualização de sua avaliação patrimonial através de uma geografia das emoções baseada no passeio, no reconhecimento, na identidade e na memória de seus habitantes, incorporando-se às classificações atuais baseadas na arquitetura das coisas já construídas.

Palavras chave: patrimônio imaterial, Festa dos Pátios de Córdoba, paisagem interior, emoções, lugar da memória. 